\title{
Why the Road to Unification Likely Goes through Krogdahl's Relativity
}

\author{
Eugene Terry Tatum \\ 760 Campbell Ln., Bowling Green, KY, USA \\ Email: ett@twc.com
}

How to cite this paper: Tatum, E.T. (2017) Why the Road to Unification Likely Goes through Krogdahl's Relativity. Journal of Modern Physics, 8, 2096-2103. https://doi.org/10.4236/jmp.2017.813128

Received: October 24, 2017

Accepted: December 4, 2017

Published: December 8, 2017

Copyright $\odot 2017$ by author and Scientific Research Publishing Inc. This work is licensed under the Creative Commons Attribution International License (CC BY 4.0).

http://creativecommons.org/licenses/by/4.0/

\begin{abstract}
Deriving an acceptable quantum field theory of gravitation from general relativity has eluded some of the best scientific thinkers. It is gradually becoming more apparent that general relativity's classical assumptions are simply incompatible with quantum mechanics. For instance, simultaneous certainty of the location and momentum of any moving body, regardless of size, is a fundamental feature of general relativity. And yet, special relativity and quantum mechanics (thru Heisenberg's uncertainty) reject the very notion of simultaneity. Since special relativity is already fully integrated into quantum field theory concerning the other forces of nature, were it possible to remove the confounding smoothly curved space-time fabric of general relativity and replace it in the form of a new and improved Lorentz-invariant (flat space-time) gravitational theory, final unification might well be achievable. This brief review paper further informs the reader as to why Krogdahl's recent Lorentz-invariant relativity model of gravitation improves on general relativity, thus providing a deeper understanding of black holes, the cosmological flatness problem and dark energy. Most importantly, since the smoothly curved space-time of general relativity may well have been the road block to unification, Krogdahl's flat space-time model is predicted to lead to an acceptable quantum theory of gravitation (i.e., "quantum gravity") and unification (i.e., a so-called "theory of everything").
\end{abstract}

\section{Keywords}

Cosmological Theory, General Relativity, Flatness Problem, Black Holes, Dark Energy, Cosmological Constant Problem, Krogdahl's Relativity

\section{Introduction and Background}

Despite valiant efforts over the past 80 years, all attempts to integrate Einstein's 
gravitational field theory (general relativity) into a unified quantum field theory have failed miserably. The precise reason for this has been a subject of much debate. However, the majority opinion to this point is that the fault lies within general relativity and not within quantum mechanics. Quantum mechanics, in the form of a quantum field theory, has already successfully incorporated the particle (as wave) dynamic fields of electromagnetic, weak and strong nuclear forces, and has proven itself over a wide range of energies. General relativity, on the other hand, has primarily proven itself to be relevant and accurate at relatively low energies and on relatively large scales. Given the exceedingly weak nature of gravity with respect to the other forces, general relativity has not, to date, been proven on scales much smaller than approximately one micron. To be fair, quantum mechanical applications on large scales are also largely unproven, with the recent exception being proof of quantum nonlocality [1]. A theoretical application of quantum mechanics to black holes, in the form of Hawking radiation, is unmeasurable and therefore unproven.

Since both theories (general relativity and quantum mechanics) make accurate predications within certain scales of energy and size, one may well ask why consolidation to a single unified field theory incorporating all of the forces of nature would seem necessary. The answer, of course, has to do with making accurate physical predictions for gravitating bodies of enormously high energy density which are compacted to such a degree that quantum effects become hugely important. These questions are most relevant with respect to an understanding of black holes and the extreme conditions of the early universe. So long as general relativity and quantum mechanics are incompatible with respect to an understanding of black holes and the early universe, we will never have the most knowledge potentially achievable of how the universe operates.

With regard to the question of why general relativity and quantum mechanics are so incompatible, it is useful to consider their important differences. The most fundamental and obvious difference is the smooth and continuous nature of general relativity space-time versus the obligate particulate nature of the quantum world. This may be a direct consequence of Einstein's starting assumptions of gravity as equivalent to acceleration and (later) isotropism and homogeneity (with regard to a cosmological model). These are classical notions with respect to the theory of quantum mechanics which came later. For a body of any size, including the smallest particle, in motion along the geodesics of general relativity one could, theoretically, have simultaneous certainty of location and momentum for all time, whereas Heisenberg's uncertainty principle precludes any such simultaneous certainty in quantum mechanics. And yet, for the sake of this discussion, it is noteworthy that notions of simultaneity are also dismissed in special relativity. So, while the most fundamental difference between general relativity and quantum mechanics makes the two theories incompatible from the start, it is important to differentiate special relativity (with its Lorentz invariance) from general relativity in this respect. Einstein's starting assumptions for 
general relativity might not necessarily be required in taking an alternate route from special relativity to a new and improved theory of gravitation, one which might be completely compatible with quantum mechanics. This notion becomes particularly relevant when considering that special relativity has already been integrated into a quantum field theory of electromagnetic, weak and strong nuclear forces.

When we ask quantum field theorists why any sort of translation between general relativity and quantum mechanics appears to be impossible, the answer generally has to do with the fact that general relativity is "non-renormalizable" [2]. This means, in layman's terms, that the infinities created in a smoothly curved space-time background cannot be ignored in developing general relativistic quantum field equations. Attempts to renormalize the infinities created simply give wildly inaccurate and nonsensical answers. Here's the key point: Since special relativity is fully integrated into quantum field theory, were it possible to remove the confounding smoothly curved space-time fabric of general relativity and replace it in the form of a new and improved Lorentz-invariant gravitational theory, final unification might well be achievable. We would then have a "quantum gravity" theory useful for understanding the quantum structure of black holes and the early universe. Other attempts at unification, such as string theory, would then become unnecessary.

It must first be conceded that general relativity is extremely accurate with respect to the canonical tests of gravitation, including:

1) Mercury's advance of perihelion

2) Solar light bending

3) Gravitational redshift

4) Echo delay of sun-grazing light beams

5) Gravity waves

6) Extreme mass densities of galactic centers

7) A mathematical model of dark energy observations

For any gravity theory to be an improvement on general relativity it would not only need to be as accurate concerning these canonical tests of gravitation, but would also have to satisfactorily address some of the ongoing problems pertaining to general relativity [3] [4]. These include:

1) Black holes and the early universe. The "singularities" produced by general relativity's infinitely curved space-time within black holes and the presumed beginning state of certain cosmological models are certainly problematic. Even Einstein had great difficulty with the notion of infinite mass-energy density. Theories of loop quantum gravity are, in fact, predicated on avoiding the seemingly nonsensical infinite singularity.

2) The "cosmological flatness problem"

3) Dark energy observations seemingly implying cosmic acceleration and the need for a non-zero cosmological constant.

4) The complete incompatibility between general relativity and the supremely 
accurate special-relativity-compatible quantum field theory incorporating electromagnetic, weak and strong nuclear forces.

Until quite recently, there have been no Lorentz-invariant theories of gravitation which have met the above criteria, although gradual improvements [5] [6] over the course of time have demonstrated that this has been an active field of investigation. Fortunately, we now have the little-noticed mathematical formulation proposed by W.A. Krogdahl, first published on arXiv.org in 2004 and revised and published in final form in 2006 [3]. It is this author's assertion that Krogdahls formulation, hereafter referred to as Krogdahls relativity, could well be the Lorentz-invariant improvement on general relativity which quantum field theorists have been looking for.

\section{Krogdahl's Relativity}

Before delving into Krogdahl's special relativistic approach to gravitation, it is first useful to know something about his background in physics and relativity in general. Wasley Krogdahl was Chandrasekhar's third Ph.D graduate (1942). Chandrasekhar was famously selective about his Ph.D candidates. They had to be particularly skilled relativists and top-rank mathematicians. Krogdahl eventually had a decades long career as a professor of Astronomy and Physics at University of Kentucky and was generally well-liked by his students. He had a gift for explaining difficult concepts in understandable terms. Late in life, as Professor Emeritus, an idea came to him about a path between special relativity and a complete relativistic theory of gravitation which would be an improvement on Einstein's path.

While Einstein had developed general relativity based upon a gravity-asacceleration equivalence principle, Krogdahl saw a better way by simply correcting the Newtonian model of gravity using special relativity's mass-energy equivalence. He started by incorporating mass-energy equivalence into Newtonian gravitational work functions, using the resulting principle that gravitational mass cannot be regarded as a fixed quantity while gravitational potential energy increases or decreases. Classical (Newtonian) gravitational equations, appropriately corrected by mass-energy equivalence, were then converted into an energy-momentum 4-vector and then conservation of energy equations were converted into Lagrange's equations.

The end result of Krogdahl's new relativistic approach was not a curved spacetime gravitational theory, with its associated complex differential geometry, but rather an equally accurate and more mathematically accessible Lorentz-invariant flat space-time gravitational theory. He published this mathematical model late in life in 2004 (first draft) and 2006 (final revision) on arXiv.org under the title, "Cosmology in Flat Space-Time" [3].

As for its accuracy with regard to the canonical tests of gravity listed above, KrogdahP s relativity is a match for general relativity. Readers can prove this for themselves by studying these proofs in pages 12-25 of Krogdahl's paper. More 
importantly, with respect to being an improvement on general relativity, KrogdahPs relativity differs with general relativity in terms of astrophysical observational predictions in three important realms. black holes, global space-time curvature, and the presumed nature of dark energy.

With respect to black holes, Krogdahl's development of gravitational redshift is perhaps most relevant. He used his equations and the law of conservation of energy to track the kinetic and potential energies of a photon moving upward from the base of a gravity well, including what we currently call a black hole. He showed that, for a photon moving upward from the base of a gravity well,

$$
V=V_{0} e^{x}
$$

where $v$ is the initial frequency at a point within the gravity well and $v_{0}$ is the "proper frequency," defined operationally as the frequency of the photon at infinite distance from the base of the gravity well (i.e., at zero gravity). Symbol $x$ represents $\left(G M / c^{2} r\right)$, pertaining to the gravitating body of interest, where distance $r$ is measured from the base of the gravity well in the upward direction to the initial frequency $v$ location. Notice that all values in the exponent are positive by this convention, so that the "proper frequency" $v_{0}$, much like proper time $t_{0}$ in special relativity, will always have the smaller value in comparison to any frequency $v$ deeper in the gravity well. The resulting frequency redshift as the photon moves upwards within the gravity well (i.e., away from the gravitating body) is the gravitational redshift.

This is a handy formula, indeed, particularly with respect to showing that no gravitating body, no matter how massive, can hold onto photons directed outward ("upward") from that body. As Krogdahl puts it, “... for any positive frequency $v$ and distance $r$, it follows that $v_{0}$ is greater than zero. Therefore, a photon would be able to escape, even from a very compact finite mass, no matter how great its surface gravity. In other words, this implies the non-existence of black holes."

Since both gravity theories (general relativity and Krogdahl's relativity) are diametrically opposed on the issue of whether there is thermal radiation emission from black holes, Sagittarius $\mathrm{A}^{*}(\mathrm{SgA})$, the supermassive black hole (SMBH) of our own Milky Way galaxy, presents an excellent testing ground. While it may not, at present, be possible to confirm by direct observation whether SgA's thermal radiation spectrum observed at Earth to be peaking at $230 \mathrm{GHz}(1.3 \mathrm{~mm}$ wavelength) originates from inner accretion disc electrons or the central black hole, Krogdahl's gravitational redshift equation offers an opportunity to test his approach, using our SgA observations.

The SgA mass and its distance from Earth can be plugged into his equation to calculate the expected gravitational redshift from SgA to Earth. In this respect, his corresponding logarithmic derivative formula,

$$
R=-G M(d r) / c^{2} r^{2}
$$

is especially useful for determining the magnitude of the gravitational redshift $\mathrm{R}$, 
using $\mathrm{r}$ equal to the $1.17626 \times 10^{10} \mathrm{~m}$ horizon distance from the geometric center of the SgA black hole, and (dr) as delta $r$ equal to the SgA-to-Earth distance of $2.47 \times 10^{20} \mathrm{~m}$. GM pertains to $\mathrm{SgA}$, where mass $\mathrm{M}$ has been estimated to be approximately $7.92 \times 10^{36} \mathrm{~kg}$ [7]. Since we use Earth as the point of observation, it is useful to remember that Earth is so far from any SMBH object of interest, including SgA, that we can treat Earth as effectively at zero gravity (i.e., defining the $v_{0}$ observation). The calculated magnitude of the redshift can then be applied to the observed peak thermal emission frequency of $230 \mathrm{GHz}$ ( $1.3 \mathrm{~mm}$ wavelength) in order to derive an initial frequency $v$ at the black hole horizon.

As outlined by this author in a paper currently in production, the observed $230 \mathrm{GHz}$ peaked thermal spectrum pinpointed to a 37 microarcsecond diameter ("event-horizon-scale") region [8] "in the immediate vicinity of the black hole" [9] appears to be consistent with black body radiation from the black hole itself! It directly overlies the geometric center of the black hole, as opposed to most accretion disc flows and jets (which also typically emit broad, non-thermal spectra). For a variety of reasons, this cannot be black hole Hawking radiation. However, using Equation (2) above, this peaked thermal spectrum is found to be an excellent fit for gamma-ray black body radiation with a peak frequency of $2.41 \times 10^{21} \mathrm{~Hz}$ which is redshifted $10.5 \mathrm{GHz}$ to our observed frequency of 230 $G H z$. This proposed black body radiation spectrum would correlate, by Wein's distribution law, to a temperature of $4.1 \times 10^{10} \mathrm{~K}$, which fits nicely with a derived minimum intrinsic brightness temperature of $2 \times 10^{10} \mathrm{~K}$ from direct observations [8]. If ultimately supported by pending VLBI studies at the highest achievable resolution, this would be a direct violation of general relativity and demonstrate the superiority of Krogdahls relativity approach as it pertains to black holes.

With respect to the general relativity problem pertaining to "singularities," Krogdahl's equations do not include the Schwarzschild metric of general relativity, wherein one of the terms has a denominator of $\left(1-2 \mathrm{GM} / \mathrm{c}^{2} \mathrm{r}\right)$ which leads to the seemingly nonsensical result (i.e., division by zero and the infinite singularity) should the astrophysical or cosmological body have a radius $r$ equal to $2 \mathrm{GM} / \mathrm{c}^{2}$.

So, while KrogdahP s relativity does not imply that the compact astrophysical bodies we refer to as black holes do not exist, they simply do not appear to require a singularity of infinite mass-energy density in Krogdahls mathematical model, and they would be expected to emit black body radiation. It is speculated by this author that black body radiation from a black hole could be a well-disguised phenomenon in most astronomical observations, for a variety of reasons, including: extremely small angular size; issues concerning the luminosity and orientation of the accretion disc and jets; confusion with other radiating bodies; difficulty observing microlensing phenomena; and the refusal of diehard general relativists to even entertain the possibility. However, most importantly, this black hole black body radiation would be gravitationally redshifted to such an extreme degree that it could easily be confused with thermal radiation originating from much 
less gravitationally redshifted sources at various distances between the observer and the black hole, including the accretion disc, jets, hot dust, etc.

As for how Krogdahl's relativity provides explanations for the "cosmological flatness problem" and the recent observations indicative of dark energy, the reader is referred to this author's paper entitled, "Why Krogdahl's Flat SpaceTime Cosmology is Superior to General Relativity" published in this special issue of Journal of Modern Physics.

Finally, a brief word about the potentially confusing topic of cosmological redshift in general relativity versus cosmological redshift in Krogdahl's relativity is in order. Although cosmological redshift is evidence of cosmic expansion, there is no observable discriminator between the "stretching" space-time of general relativity and the Lorentz-invariant relativistic Doppler redshift (stretching wavelength) of flat space-time cosmology. Both cosmologies give entirely different explanations for the redshift, but rely on the same relativistic formula, $s=(z+1)=[(1+v / c) /(1-v / c)]^{1 / 2}$. For instance, this formula was used successfully in this author's first two papers on the heuristic "Flat Space Cosmology" (FSC) model [10] [11].

\section{Conclusion}

Special relativity is already fully incorporated into the supremely accurate quantum field theory for electromagnetic, weak and strong nuclear forces. Lorentzinvariance does not create the incompatibility with quantum mechanics that is clearly evident with general relativity. It should be obvious from the foregoing discussion that a Lorentz-invariant gravitational theory which not only passes the canonical tests of gravitation but also improves on general relativity with respect to the treatment of black holes, the cosmological flatness problem, and dark energy observations could well be the path to a final unification theory of all of the forces of nature. In these respects, Krogdahl's relativity appears to be superior to general relativity as a theory of gravitation.

\section{References}

[1] Erven, C. Meyer-Scott, E., Lavoie, J., et.al. (2014) Nature Photonics, 8, 292-296. https://doi.org/10.1038/nphoton.2014.50

[2] Shomer, A. (2017) A Pedogogical Explanation for the Non-Renormalizability of Gravity. arXiv:0709.3555v2 [hep-th]

[3] Krogdahl, W.S. (2006) Cosmology in Flat Space-Time. arXiv:gr-qc/0402016

[4] Krogdahl, W.S. A Critique of General Relativity. http://arxiv.org/pdf/0711.1145.pdf

[5] Scharf, G. (2015) Against Geometry: Nonstandard General Relativity. arXiv:1208.3749v5 [gr-qc].

[6] Behera, H. and Naik, P. (2003) A Flat Space-Time Relativistic Explanation for the Perihelion Advance of Mercury. arXiv:astro-ph/0306611v1.

[7] Boehle, A., Ghez, R., Schodel, R., et.al. (2016) An Improved Distance and Mass Estimate for Sgr $\mathrm{A}^{\star}$ from a Multistar Orbit Analysis. ApJ, 830.

https://arxiv.org/abs/1607.05726v1 
[8] Doeleman, S., et.al. (2008) Nature, 455, 78-80. https://doi.org/10.1038/nature07245

[9] Dexter, J. and Fragile, P. (2012) Monthly Notices of the Royal Astronomical Society, 1-17.

[10] Tatum, E.T., Seshavatharam, U.V.S. and Lakshminarayana, S. (2015) International Journal of Astronomy and Astrophysics, 5, 116-124. https://doi.org/10.4236/ijaa.2015.52015

[11] Tatum, E.T., Seshavatharam, U.V.S. and Lakshminarayana, S. (2015) Journal of Applied Physical Science International, 4, 18-26. 